\title{
Abnormal response of left ventricular systolic function to submaximal exercise in post-partial left ventriculotomy patients
}

A.H. Herdy ${ }^{1}$, P. Moritz ${ }^{1}$, A.V. Assis ${ }^{1}$, F. Ribeiro ${ }^{1}$, J. Collaço ${ }^{1}$ and J.P. Ribeiro ${ }^{2,3}$
${ }^{1}$ Instituto de Cardiologia de Santa Catarina, Florianópolis, SC, Brasil ${ }^{2}$ Serviço de Cardiologia, Hospital de Clínicas de Porto Alegre, ${ }^{3}$ Departamento de Medicina Interna, Faculdade de Medicina, Universidade Federal do Rio Grande do Sul, Porto Alegre, RS, Brasil
Correspondence

J.P. Ribeiro

Serviço de Cardiologia

Hospital de Clínicas de Porto Alegre

Rua Ramiro Barcelos, 2350

90035-007 Porto Alegre, RS

Brasil

Fax: +55-51-316-8657

E-mail: jpribeiro@cpovo.net

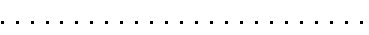

Received September 30, 2005

Accepted March 27, 2006

\begin{abstract}
Patients with heart failure who have undergone partial left ventriculotomy improve resting left ventricular systolic function, but have limited functional capacity. We studied systolic and diastolic left ventricular function at rest and during submaximal exercise in patients with previous partial left ventriculotomy and in patients with heart failure who had not been operated, matched for maximal and submaximal exercise capacity. Nine patients with heart failure previously submitted to partial left ventriculotomy were compared with 9 patients with heart failure who had not been operated. All patients performed a cardiopulmonary exercise test with measurement of peak oxygen uptake and anaerobic threshold. Radionuclide left ventriculography was performed to analyze ejection fraction and peak filling rate at rest and during exercise at the intensity corresponding to the anaerobic threshold. Groups presented similar exercise capacity evaluated by peak oxygen uptake and at anaerobic threshold. Maximal heart rate was lower in the partial ventriculotomy group compared to the heart failure group $(119 \pm 20$ vs $149 \pm 21 \mathrm{bpm} ; \mathrm{P}<0.05)$. Ejection fraction at rest was higher in the partial ventriculotomy group as compared to the heart failure group $(41 \pm 12$ vs $32 \pm 9 \%$; $\mathrm{P}<0.0125)$; however, ejection fraction increased from rest to anaerobic threshold only in the heart failure group (partial ventriculotomy $=44 \pm 17 \%$; $\mathrm{P}=$ nonsignificant $v s$ rest; heart failure $=39 \pm 11 \%$; $\mathrm{P}<0.0125$ vs rest; $\mathrm{P}<$ 0.0125 vs change in the partial ventriculotomy group). Peak filling rate was similar at rest and increased similarly in both groups at the anaerobic threshold intensity (partial ventriculotomy $=2.28 \pm 0.55$ $\mathrm{EDV} / \mathrm{s}$; heart failure $=2.52 \pm 1.07 \mathrm{EDV} / \mathrm{s} ; \mathrm{P}<0.0125 ; \mathrm{P}>0.05 v s$ change in partial ventriculotomy group). The abnormal responses demonstrated here may contribute to the limited exercise capacity of patients with partial left ventriculotomy despite the improvement in resting left ventricular systolic function.
\end{abstract}

Key words

- Heart failure

- Radionuclide

ventriculography

- Anaerobic threshold

- Left ventricular diastolic function

- Left ventricular ejection fraction 


\section{Introduction}

Heart transplantation is considered to be the treatment of choice for patients with endstage heart failure secondary to left ventricular systolic dysfunction but, due to the limited availability of donors and certain contraindications, other surgical procedures have been proposed for the treatment of these patients (1). Partial left ventriculotomy is a surgical procedure that is based on the law of Laplace, by which the circumferential wall stress is directly proportional to intraventricular pressure and radius, and inversely proportional to wall thickness (2). Accordingly, the surgical excision of part of the free wall of the left ventricle, with reduction of the radius, could improve wall stress with consequent improvement in function. Early reports using this procedure demonstrated benefits in symptoms as well as improvement in resting left ventricular systolic function (2-4). However, other studies indicated elevated in-hospital and long-term mortality rates (5), resulting in a marked reduction in the use of this procedure. However, a subgroup of survivors improves clinically, with enhancement of quality of life, despite the persistence of limited exercise capacity.

Little is known about the mechanisms responsible for the limited exercise capacity despite the improvement in resting left ventricular function in post-left ventriculotomy patients. In normal individuals and in patients with heart failure, indices of left ventricular systolic and diastolic function increase from rest to submaximal exercise $(6,7)$. However, there are no studies in which the response of left ventricular systolic and diastolic function to exercise was evaluated in post-partial left ventriculotomy patients. In order to better understand the mechanisms associated with this limited exercise capacity, we studied the systolic and diastolic left ventricular function at rest and during submaximal exercise in patients with partial left ventriculotomy and in patients with heart failure who had not been operated, matched for maximal and submaximal exercise capacity.

\section{Patients and Methods}

\section{Study patients}

Patients were recruited from the Heart Institute of the Santa Catarina Cardiomyopathy Outpatient Clinic. Nine patients had been previously submitted to partial left ventriculotomy and were studied 3 to 18 months after surgery, and 9 patients had not been submitted to surgical intervention. Inclusion criteria were stable condition, being in New York Heart Association (NYHA) functional class II or III, for at least one month, and no contraindications for maximal exercise testing. The following were exclusion criteria: presence of arrhythmia or a pacemaker, NYHA functional class IV, significant pulmonary disease, uncorrected valvular heart disease, and exercise-induced myocardial ischemia. The investigation conformed with the principles outlined in the Declaration of Helsinki, the protocol was approved by the Committee for Ethics in Research of the Heart Institute of Santa Catarina, and all patients signed an informed consent form.

\section{Study protocol}

All patients were submitted to a maximal cardiopulmonary exercise test to determine the heart rate corresponding to the anaerobic threshold and of the peak oxygen uptake $\left(\mathrm{VO}_{2}\right.$ peak). Approximately one week later, resting and exercise radioisotopic ventriculography was performed. The exercise radioisotopic ventriculography was performed at the intensity corresponding to the heart rate at the anaerobic threshold or, if the anaerobic threshold could not be determined due to oscillatory ventilation (8), at $70 \%$ of peak heart rate. 


\section{Cardiopulmonary exercise testing}

Patients were monitored continuously with a $\mathrm{CM}_{5}$ electrocardiographic lead using an electrocardiograph (Vmax 229; Sensor Medics Corp., Yorba Linda, CA, USA) while performing an increasingly stepped exercise test on an electrically braked cycle ergometer (Vmax 229, Sensor Medics). After 4 min of unloaded cycling, the power output was increased using a continuous ramp protocol at a rate of $10 \mathrm{~W} / \mathrm{min}$ until the patients could not maintain the pedaling rate of 60 rpm. During the test, patients breathed through a previously calibrated commercial gas exchange analysis system (Vmax 229, Sensor Medics). $\mathrm{VO}_{2}$ peak was identified as the highest $\mathrm{VO}_{2}$ maintained for $30 \mathrm{~s}$. The anaerobic threshold was determined by the V-slope method (9).

\section{Radioisotopic left ventriculography}

Left ventricular systolic and diastolic function was evaluated at rest and at the intensity corresponding to the anaerobic threshold in the sitting position, with the chest inclined at about 35 degrees and with the collimator positioned at a left anterior oblique projection. Image acquisition was performed after in vivo red cell labeling with Tc-99m pertechnetate, using a Siemens Orbiter camera with a ZLC-DIGITRAC 75 detector (Siemens, Hoffman Estates, IL, USA). Images were photographed with a Micro Dot Imager (Siemens) and recorded in a Microdelta computer (Siemens). Left ventricular ejection fraction was calculated as left ventricular end-diastolic counts minus left ventricular end-systolic counts, divided by end-diastolic counts, always subtracting periventricular radiation. Peak filling rate was calculated by a series of subtractions of each successive frame in the part of the left ventricular function curve corresponding to the rapid filling period, and identified as the peak rate of filling normalized by the end- diastolic counts $(6,10)$.

\section{Statistical analysis}

Descriptive data are presented as mean \pm SD. Continuous variables were compared by the Student $t$-test for unpaired samples. Proportions were compared by the Fischer exact test. Differences were considered to be significant when $\mathrm{P}<0.05$. The response of left ventricular function from rest to anaerobic threshold was evaluated by the Wilcoxon sign rank test. To compensate for multiple comparisons, the Bonferroni correction was applied and a $\mathrm{P}<0.0125$ level was required.

\section{Results}

\section{Clinical characteristics}

Table 1 demonstrates that the age and anthropometric characteristics of post-left ventriculotomy patients were similar when compared to those of heart failure patients. Etiology of heart failure as well as the use of medication, including beta-blockers, were not significantly different between the two groups. Considering all 18 patients recruited, etiology of heart failure was ischemic in 9 patients, primary cardiomyopathy in 6 , corrected valvular heart disease in 2, and alcoholic cardiomyopathy in 1 patient. All patients were taking furosemide, 17 patients were taking angiotensin converting inhibitors or angiotensin receptor antagonists, 11 patients were taking spironolactone, 9 patients were on amiodarone, and 8 patients were taking beta-blockers. The groups had similar echocardiographically determined left ventricular end-diastolic dimension and belonged to the same NYHA functional class (Table 1).

\section{Cardiopulmonary exercise testing}

The groups were well matched for maximal as well as submaximal exercise capacity 
as determined by $\mathrm{VO}_{2}$ peak, peak power output, and $\mathrm{VO}_{2}$ at anaerobic threshold (Table 1). Respiratory exchange ratio at peak exercise and at anaerobic threshold was also similar. Resting heart rate and heart rate at anaerobic threshold tended to be higher in the heart failure group. At peak exercise, heart rate was significantly higher in the heart failure group.

\section{Radionuclide left ventriculography}

Figure 1 presents individual as well as

Table 1. Characteristics and results of cardiopulmonary exercise testing of post-left ventriculotomy patients and patients with heart failure.

\begin{tabular}{|c|c|c|}
\hline & Post-left ventriculotomy & Heart failure \\
\hline Age (years) & $55 \pm 5$ & $49 \pm 12$ \\
\hline Gender (male) & 6 & 6 \\
\hline Weight $(\mathrm{kg})$ & $68 \pm 9$ & $69 \pm 10$ \\
\hline Height $(\mathrm{cm})$ & $168 \pm 8$ & $164 \pm 7$ \\
\hline \multicolumn{3}{|l|}{ Medication } \\
\hline Digoxin & 7 & 9 \\
\hline Furosemide & 9 & 9 \\
\hline ACEi/ARAII & 8 & 9 \\
\hline Beta-blockers & 3 & 5 \\
\hline Spironolactone & 5 & 6 \\
\hline Amiodarone & 5 & 4 \\
\hline \multicolumn{3}{|l|}{ Etiology } \\
\hline Coronary artery disease & 4 & 5 \\
\hline Dilated cardiomyopathy & 3 & 3 \\
\hline Operated valvular heart disease & 2 & 0 \\
\hline Alcoholic cardiomyopathy & 0 & 1 \\
\hline $\begin{array}{l}\text { Echocardiography-determined left } \\
\text { ventricular end-diastolic dimension }(\mathrm{cm})\end{array}$ & $7.0 \pm 1.0$ & $7.0 \pm 0.4$ \\
\hline NYHA functional class & $2.0 \pm 0.5$ & $2.0 \pm 0.5$ \\
\hline $\mathrm{VO}_{2}$ peak $\left(\mathrm{mL} \mathrm{kg}^{-1} \mathrm{~min}^{-1}\right)$ & $13.1 \pm 3.3$ & $14.1 \pm 3.6$ \\
\hline Peak power output (W) & $92 \pm 34$ & $77 \pm 31$ \\
\hline Peak respiratory exchange ratio & $1.06 \pm 0.03$ & $1.05 \pm 0.03$ \\
\hline $\mathrm{VO}_{2}$ at anaerobic threshold $\left(\mathrm{mL} \mathrm{kg}{ }^{-1} \mathrm{~min}^{-1}\right)$ & $7.9 \pm 1.3$ & $8.5 \pm 1.6$ \\
\hline $\begin{array}{l}\text { Respiratory exchange ratio at } \\
\text { anaerobic threshold }\end{array}$ & $0.86 \pm 0.03$ & $0.88 \pm 0.05$ \\
\hline Resting heart rate (bpm) & $74 \pm 17$ & $87 \pm 17$ \\
\hline Heart rate at anaerobic threshold (bpm) & $95 \pm 20$ & $112 \pm 20$ \\
\hline Peak heart rate (bpm) & $119 \pm 20$ & $149 \pm 21^{*}$ \\
\hline
\end{tabular}

Data are reported as mean $\pm S D$ or as number of patients ( $N=9$ in each group). ACEi = angiotensin converting enzyme inhibitors; ARAll = angiotensin II receptor antagonists; $\mathrm{NYHA}=$ New York Heart Association; $\mathrm{VO}_{2}=$ oxygen uptake.

${ }^{*} \mathrm{P}<0.05$ compared to the post-left ventriculotomy group (Student $t$-test for unpaired samples). mean $( \pm \mathrm{SD})$ values for systolic and diastolic left ventricular function at rest and at the exercise intensity corresponding to the anaerobic threshold. Resting left ventricular ejection fraction was significantly higher for post-left ventriculotomy patients compared to heart failure patients (left ventriculotomy: $41 \pm 12 \%$ vs heart failure: $32 \pm 9 \%$; $\mathrm{P}<$ 0.0125). Ejection fraction increased from rest to the anaerobic threshold only in the heart failure group (partial ventriculotomy: $44 \pm 17 \%$; $\mathrm{P}=$ non-significant $v s$ rest; heart failure: $39 \pm 11 \% ; \mathrm{P}<0.0125$ vs rest; $\mathrm{P}<$ $0.0125 v s$ change in the partial ventriculotomy group). Resting peak filling rate was similar in the two groups (partial ventriculotomy: $1.41 \pm 0.55 \mathrm{EDV} / \mathrm{s}$; heart failure: 1.39 $\pm 0.55 \mathrm{EDV} / \mathrm{s} ; \mathrm{P}>0.05)$ and increased significantly to the anaerobic threshold intensity in both groups (partial ventriculotomy: $2.28 \pm 0.55 \mathrm{EDV} / \mathrm{s} ; \mathrm{P}<0.0125$ vs rest; heart failure: $2.52 \pm 1.07 \mathrm{EDV} / \mathrm{s} ; \mathrm{P}<0.0125$ vs rest; $\mathrm{P}>0.05$ vs change in partial ventriculotomy group).

\section{Discussion}

In 1997, partial left ventriculotomy emerged as a surgical alternative for endstage heart failure patients who were not candidates for heart transplantation (2). Despite high operative and in-hospital mortality rates, early reports generated optimism in the surgical community $(3,4,11)$, mainly due to improvement of resting left ventricular ejection fraction and functional class, as well as due to reduction in left ventricular volume $(3,12-14)$. Despite the fact that some groups have reported poor long-term results (15), many survivors of partial-left ventriculotomy improve functional status, but may not normalize functional capacity. To our knowledge, the present report is the first to consider the responses of left ventricular systolic and diastolic function to exercise in post-left ventriculotomy patients.

The group of post-left ventriculotomy 
patients and the group of patients not previously operated belonged to a similar NYHA functional class and had similar $\mathrm{VO}_{2}$ peak, peak power output, and $\mathrm{VO}_{2}$ at anaerobic threshold, indicating that they were well matched for maximal as well as submaximal exercise capacity, which allowed us to investigate possible limiting factors in exercise capacity for these groups. Despite a similar echocardiographically determined left ventricular end-diastolic dimension, postleft ventriculotomy patients had a significantly higher resting left ventricular ejection fraction, which is consistent with some previous observations (12-14). Some investigators have also suggested that the improvement in resting left ventricular systolic function is associated with postoperative reduction of wall stress (13-14).

Although our heart failure group presented lower resting ejection fraction, they were able to improve left ventricular systolic function at the anaerobic threshold. Previous studies on heart failure patients as well as normal subjects reported significant increase in left ventricular ejection fraction at the anaerobic threshold intensity, with the exception of those who had exercise-induced myocardial ischemia $(6,7,16,17)$. Thus, our heart failure patients presented an appropriate left ventricular systolic function response to exercise, while our post-left ventriculotomy patients did not increase left ventricular ejection fraction at the anaerobic threshold intensity. This abnormal left ventricular response of systolic function may contribute to the limited exercise capacity of post-leftventriculotomy patients.

There are very limited data on the response of left ventricular diastolic function to exercise in heart failure patients, but it is known that normal subjects show a significant increase in left ventricular peak filling rate from rest to the anaerobic threshold intensity (6). At rest, peak filling rate was similar in our two groups. Likewise, both our post-left ventriculotomy patients and our heart failure patients presented similar in-
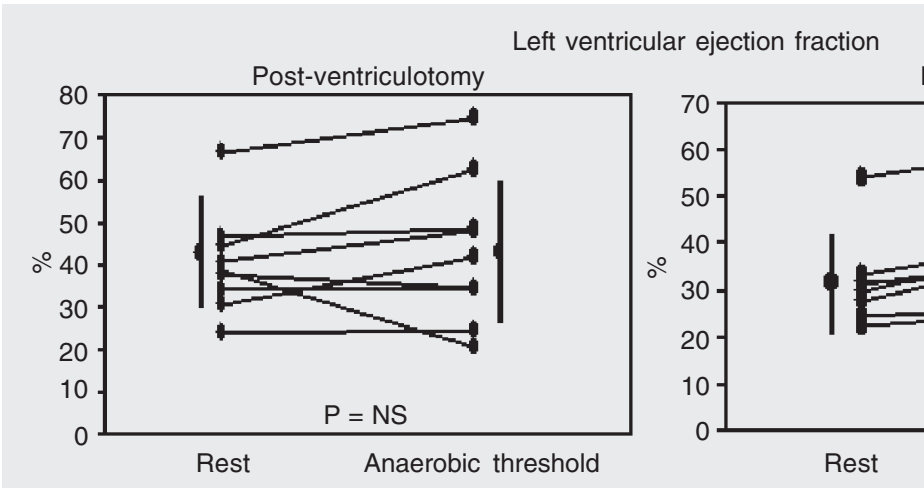

Heart failure

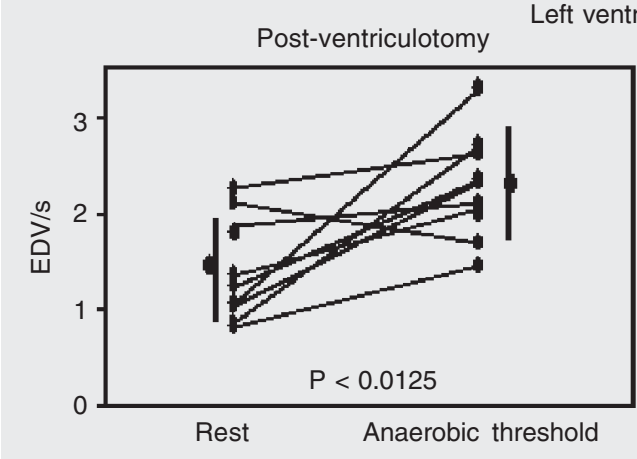

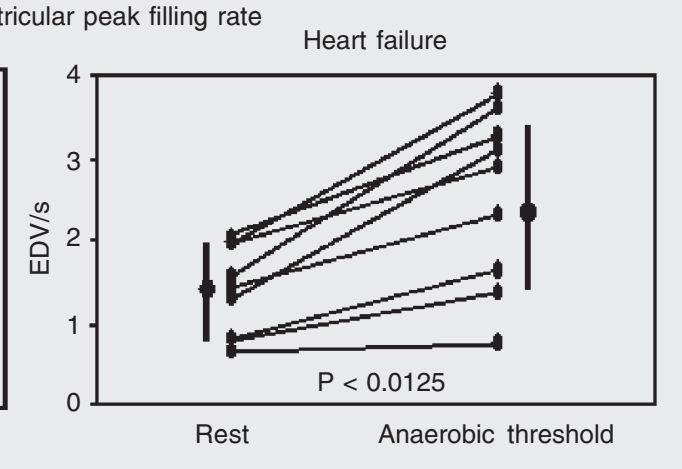

Figure 1. Left ventricular ejection fraction (upper panels) and left ventricular peak filling rate (lower panels) at rest and at the anaerobic threshold intensity in post-left ventriculotomy (left panels) and heart failure patients (right panels). Data are reported as individual values and as mean $\pm S D$ (to the left and right of each panel). For ejection fraction, the change in the heart failure group was significantly different from the change in the post-left ventriculotomy group ( $P$ $<0.0125$, Wilcoxon sign rank test). The increase in peak filling rate was similar in the two groups). 
creases in peak filling rate from rest to the anaerobic threshold intensity, suggesting that left ventricular diastolic dysfunction is not the main determinant of the limited exercise capacity of post-left ventriculotomy patients.

One possible explanation for the limited response of systolic function to exercise in post-left ventriculotomy patients could be the late inappropriate remodeling of the left ventricle, reducing efficiency in systolic contraction. In the early postoperative period, the reduction in left ventricular volume could reduce wall stress and improve contractility, but late remodeling, to which mitral regurgitation (18) and apoptosis (19) may contribute, could result in a progressive loss of this benefit. In fact, some patients experience improved quality of life immediately after the intervention, but may show deterioration during long-term follow-up.

Colucci et al. (20) demonstrated that the chronotropic response to exercise is strongly associated with maximal exercise capacity evaluated by $\mathrm{VO}_{2}$ peak in patients with heart failure. Our post-left ventriculotomy patients had more impairment in the chronotropic response to exercise when compared to the heart failure patients. The reduction of maximal heart rate could be another mechanism contributing to the limited functional capacity of post-left ventriculotomy patients. Since chronotropic incompetence is associated with a desensitization of beta-adrenergic receptors in the sinus node (20), it is possible that partial left ventriculotomy does not improve this abnormality and patients would maintain a limited chronotropic response to exercise, further limiting functional capacity.

Post-left ventriculotomy patients present an abnormal response of left ventricular systolic function to submaximal exercise at the intensity of anaerobic threshold and a limited chronotropic response to maximal exercise. These abnormal responses may partially explain the limited functional capacity of these patients despite the improvement in resting left ventricular systolic function.

\section{References}

1. Hunt SA, Baker DW, Chin MH, Cinquegrani MP, Feldman AM, Francis GS, et al. ACC/AHA guidelines for the evaluation and management of chronic heart failure in the adult: executive summary. A report of the American College of Cardiology/American Heart Association Task Force on Practice Guidelines (Committee to Revise the 1995 Guidelines for the Evaluation and Management of Heart Failure). J Am Coll Cardiol 2001; 38: 2101-2113.

2. Batista RJ, Verde J, Nery P, Bocchino L, Takeshita N, Bhayana JN, et al. Partial left ventriculectomy to treat end-stage heart disease. Ann Thorac Surg 1997; 64: 634-638.

3. Bocchi EA, Bellotti G, Vilella de Moraes A, Bacal F, Moreira LF, Esteves-Filho A, et al. Clinical outcome after left ventricular surgical remodeling in patients with idiopathic dilated cardiomyopathy referred for heart transplantation: short terms results. Circulation 1997; 96 (Suppl II): 165-172.

4. Stolf NA, Moreira LF, Bocchi EA, Higuchi ML, Bacal F, Bellotti G, et al. Determinants of midterm outcome of partial left ventriculectomy in dilated cardiomyopathy. Ann Thorac Surg 1998; 66: 1585-1591.

5. Starling RC, McCarthy PM. Partial left ventriculectomy: sunrise or sunset? Eur J Heart Fail 1999; 1: 313-317.

6. Clausell N, Ludwig E, Narro F, Ribeiro JP. Response of left ventricular diastolic filling to graded exercise relative to the lactate threshold. Eur J Appl Physiol Occup Physiol 1993; 67: 222-225.

7. Koike A, Itoh H, Taniguchi K, Hiroe M. Detecting abnormalities in left ventricular function during exercise by respiratory measurement.
Circulation 1989; 80: 1737-1746.

8. Ribeiro JP, Knutzen A, Rocco MB, Hartley LH, Colucci WS. Periodic breathing during exercise in severe heart failure. Reversal with milrinone or cardiac transplantation. Chest 1987; 92: 555-556.

9. Beaver WL, Wasserman K, Whipp BJ. A new method for detecting anaerobic threshold by gas exchange. J Appl Physiol 1986; 60: 2020-2027.

10. Polak JF, Kemper AJ, Bianco JA, Parisi AF, Tow DE. Resting early peak diastolic filling rate: a sensitive index of myocardial dysfunction in patients with coronary artery disease. J Nucl Med 1982; 23: 471-478.

11. Moreira LF, Stolf NA, Bocchi EA, Bacal F, Giorgi MC, Parga JR, et al. Partial left ventriculectomy with mitral valve preservation in the treatment of patients with dilated cardiomyopathy. $J$ Thorac Cardiovasc Surg 1998; 115: 800-807.

12. McCarthy PM, Starling RC, Wong J, Scalia GM, Buda T, Vargo RL, et al. Early results with partial left ventriculectomy. $J$ Thorac Cardiovasc Surg 1997; 114: 755-763.

13. Gorcsan III J, Feldman AM, Kormos RL, Mandarino WA, Demetris AJ, Batista RJ. Heterogeneous immediate effects of partial left ventriculectomy on cardiac performance. Circulation 1998; 97: 839842.

14. Popovic Z, Miric M, Gradinac S, Neskovic AN, Jovovic L, Vuk L, et al. Effects of partial left ventriculectomy on left ventricular performance in patients with nonischemic dilated cardiomyopathy. J Am 
Coll Cardiol 1998; 32: 1801-1808.

15. Starling RC, McCarthy PM, Buda T, Wong J, Goormastic M, Smedira $N G$, et al. Results of partial left ventriculectomy for dilated cardiomyopathy: hemodynamic, clinical and echocardiographic observations. J Am Coll Cardiol 2000; 36: 2098-2103.

16. Port S, McEwan P, Cobb FR, Jones RH. Influence of resting left ventricular function on the left ventricular response to exercise in patients with coronary artery disease. Circulation 1981; 63: 856863.

17. Schoolmeester WL, Simpson AG, Sauerbrunn BJ, Fletcher RD. Radionuclide angiographic assessment of left ventricular function during exercise in patients with a severely reduced ejection fraction.
Am J Cardiol 1981; 47: 804-809.

18. Kawaguchi AT, Bocchino LO, Shimura S, Karamanoukian HL, Koide $\mathrm{S}$, Batista RJ. Mitral regurgitation after partial left ventriculectomy as the cause of ventricular redilatation. J Card Surg 2001; 16: 89-96.

19. Metzger M, Higuchi ML, Moreira LF, Chaves MJ, Castelli JB, Silvestre JM, et al. Relevance of apoptosis and cell proliferation for survival of patients with dilated cardiomyopathy undergoing partial left ventriculectomy. Eur J Clin Invest 2002; 32: 394-399.

20. Colucci WS, Ribeiro JP, Rocco MB, Quigg RJ, Creager MA, Marsh $\mathrm{JD}$, et al. Impaired chronotropic response to exercise in patients with congestive heart failure. Role of postsynaptic beta-adrenergic desensitization. Circulation 1989; 80: 314-323. 